\title{
BMJ Open Advance care planning for patients with advanced illnesses attending hospital outpatient clinics study: a study protocol for a randomised controlled trial
}

To cite: Rhee J, Meller A, Krysinska K, et al. Advance care planning for patients with advanced illnesses attending hospital outpatient clinics study: a study protocol for a randomised controlled trial. BMJ Open 2019;9:e023107. doi:10.1136/ bmjopen-2018-023107

- Prepublication history for this paper is available online. To view these files, please visit the journal online (http://dx.doi org/10.1136/bmjopen-2018023107).

Received 21 March 2018 Revised 21 November 2018 Accepted 28 November 2018

Check for updates

(C) Author(s) (or their employer(s)) 2019. Re-use permitted under CC BY-NC. No commercial re-use. See rights and permissions. Published by BMJ.

For numbered affiliations see end of article.

Correspondence to

Associate Professor Joel Rhee jrhee@uow.edu.au

\section{ABSTRACT}

Introduction It is unclear whether advance care planning (ACP) undertaken with patients living in the community can improve patient care and avoid unwanted interventions and hospital admissions. We have designed a randomised controlled trial (RCT) to examine if ACP undertaken with patients with advanced illnesses attending hospital outpatient clinics can reduce unplanned hospital admissions and improve patient and caregiver well-being.

Methods and analysis Pragmatic RCT involving patients from subspecialty outpatient clinics at five clinical sites in Sydney, Australia. Participants will be $\geq 18$ years screened as potentially having palliative care needs and at risk of dying in 6-12 months. The patients will be randomised to intervention or control group. Intervention group will undertake ACP discussions facilitated by a trained health professional. The control group will receive written information on ACP, representing the current standard of care. The primary outcome is the number of unplanned hospital admissions at the 6-month follow-up. Secondary outcomes include: (i) patient's health-related quality-oflife and quality of chronic disease care; (ii) caregiver's health-related quality-of-life and caregiver burden and (iii) other health outcomes including ambulance usage, emergency department presentations, hospital admissions, resuscitation attempts, intensive care unit admissions, deaths, documentation of patient wishes in patient records and audit of ACP discussions and documents. The staff's self-reported attitudes and knowledge of ACP will also be measured. The data will be collected using self-report questionnaires, hospital records audit, audit of ACP documentation and data linkage analysis. Semistructured interviews and focus group discussions with patients, caregivers and healthcare professionals will explore the acceptability and feasibility of the intervention.

Ethics and dissemination Approved by South-East Sydney Local Health District Human Research Ethics Committee and NSW Population and Health Services Research Ethics Committee. Results will be disseminated via conference presentations, journal publications, seminars and invited talks.

Trial registration number ACTRN12617000280303.
Strengths and limitations of this study

- Due to its design as a pragmatic randomised controlled trial in real-world conditions, the findings of the study are likely to be generalisable to clinical practice.

- Multiple sources of data including surveys of participants, audit of medical records, health outcomes data obtained through data linkage and qualitative data (focus groups and interviews) will allow the study to examine the effectiveness of the intervention on health outcomes, patient reported outcomes as well as its impact on health professionals and clinical services.

- The multiple sources of data will also allow triangulation of the findings, especially health outcomes.

- The sample size takes into account the significant drop-out rate expected in the study due to withdrawal and/or loss to follow-up and high mortality expected in the study patient population.

\section{INTRODUCTION}

Advance care planning (ACP) is a process of reflection, discussion and communication that enables a person to plan for their future medical treatment and other care for a time when they are not competent to make, or communicate, decisions for themselves. ${ }^{2}$ ACP can significantly improve the quality of care provided to patients with advanced illnesses, ${ }^{3}$ increase the chance of patient's wishes being known and followed, improve emotional outcomes for the patient and caregivers and increase caregiver satisfaction. ${ }^{45}$ There is also some evidence that ACP improves the patient's quality of life, ${ }^{5}$ reduces ambulance use and unplanned hospital admissions $^{6}{ }^{7}$ and contributes to reduced patient mortality. ${ }^{6}$

The importance of ACP in patient care has been widely recognised and endorsed by a large number of professional groups, specialist 
colleges, peak bodies and patient advocate groups and in diverse clinical populations. ${ }^{8}$ In Australia, the National Health and Hospitals Reform Commission ${ }^{9}$ established by the Federal Government identified the importance of ACP and recommended its implementation on a national scale. The New South Wales (NSW) Ministry of Health created a policy on ACP $;{ }^{10}$ its priority is to incorporate ACP into routine care, to educate health professionals in both conducting and responding to ACP and to improve collaboration between NSW clinical services and community and primary care health professionals.

Despite the wide recognition and endorsement, ACP is not commonly done in Australia. ${ }^{11}$ A chart audit conducted in 2006 in a large tertiary teaching hospital in Australia showed that none of 47 consecutive patients whose treatment was withdrawn in the intensive care unit had evidence of ACP. ${ }^{12}$ An older retrospective chart audit of 110 patients older than 65 years of age who died in a Sydney hospital showed the prevalence of Advance Care Plans (unclear but presumably informal documents) of $7.3 \% .{ }^{13}$ Studies from other Australian jurisdictions do not provide additional insights into the prevalence of ACP, except that it is very uncommon. ${ }^{14}$

Current evidence that ACP may lead to reduction in ambulance use and hospital admissions exists mainly in the residential aged care home setting. ${ }^{6}$ There is a lack of studies in Australia conducted in the community or outpatient settings, which examine the effect of ACP on unplanned hospital admissions or the use of acute health services. Busy emergency departments (EDs) and hospital wards are not ideal settings for ACP, and there is evidence that ACP decisions made when acutely unwell differ from decisions made when medically stable, so outpatient clinics are a better setting in which to conduct ACP. ${ }^{15}$ There is, however, lack of training and support for staff working in outpatient clinics to first identify patients who may benefit from ACP and second to facilitate ACP.

\section{OBJECTIVE}

The objective of this study is to determine whether a model of facilitated ACP intervention based in hospital outpatient clinics will reduce unplanned hospital admissions and acute health services usage, improve the quality of care for patients and their caregivers/family and result in improved understanding and uptake of ACP by health professionals.

\section{METHODS AND ANALYSIS \\ Study design and setting}

This study is a pragmatic, prospective, multisite randomised controlled trial (RCT) with two parallel groups. It is a pragmatic RCT testing the real-world effectiveness of a complex intervention aimed at embedding ACP into routine care provided by hospital outpatient clinics. The study setting is subspecialty outpatient clinics (renal, cardiac, liver, respiratory, aged care and other relevant clinics) at five clinical sites across two Local Health Districts (LHDs) in Sydney, Australia: South East Sydney LHD (Prince of Wales Hospital, Sutherland Hospital, St George Hospital, War Memorial Hospital) and Sydney LHD (Concord Hospital).

\section{Participants}

To be eligible for the study, patients must be 18 years or older and attend an outpatient clinic of participating clinical services. They must be identified as potentially having supportive and palliative care needs and be at potential risk of dying in the next 6-12 months, as determined by the Supportive and Palliative Care Indicators Tool (SPICT) ${ }^{16}$ Patients must be interested in engaging in ACP as determined by the ACP screening tool ${ }^{17}$ and be willing and able to give written informed consent. If SPICT reveals that the patient has dementia or other illnesses that may impact on the patient's decision-making capacity, then the treating medical team will determine if they have the capacity to provide informed consent. If they do not, then consent will be obtained from the person responsible (substitute decision-maker according to the hierarchy as defined by the NSW Guardianship Act 1987), together with the patient.

Patients are not eligible for the trial if they are younger than 18 years of age, are pregnant or have both appointed an enduring guardian and made an advance care directive (ACD)/plan as determined by the ACP screening tool. ${ }^{17}$ Current inpatients or permanent residents of a residential aged care facility will be excluded from the study. Patients (or if a patient lacks decision making capacity, his/her guardian or person responsible) who are unable or unwilling to provide informed consent or are unable to provide informed consent and participate in ACP conducted in English due to difficulties in understanding and speaking English, also will be excluded. Figure 1 describes the participant assessment and follow-up plan.

\section{Intervention and control}

Patients randomised to the control group will be provided written information on ACP, which represents the current standard care. The written information consists of: (1) ACP brochure produced by NSW Health, ${ }^{18}$ which contains basic information about ACP and where to get assistance if interested; (2) ACP workbook endorsed by NSW Health $;^{19}$ (3) Enduring Guardianship NSW booklet ${ }^{20}$ and (4) Statement of Values and Wishes completed on behalf of the patient by their person responsible, when the patient is unable to develop an ACD.

The intervention group will be provided the same written information on ACP as the control group; however, ACP discussions will be actively facilitated by an ACP coordinator or other trained health professional (table 1). This includes discussions with patients, caregivers, family and clinical staff, communications with other specialists, general practitioners (GPs) and other health providers involved in patient care, assistance with completing documentation and assistance with dissemination of ACP 
Identification of potential participants (SPICT)*

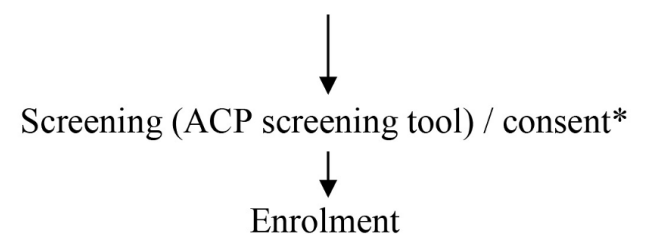

Completion by patient of baseline questionnaire

Completion by caregiver (separately consented) of baseline questionnaire

Complete Charlson Index

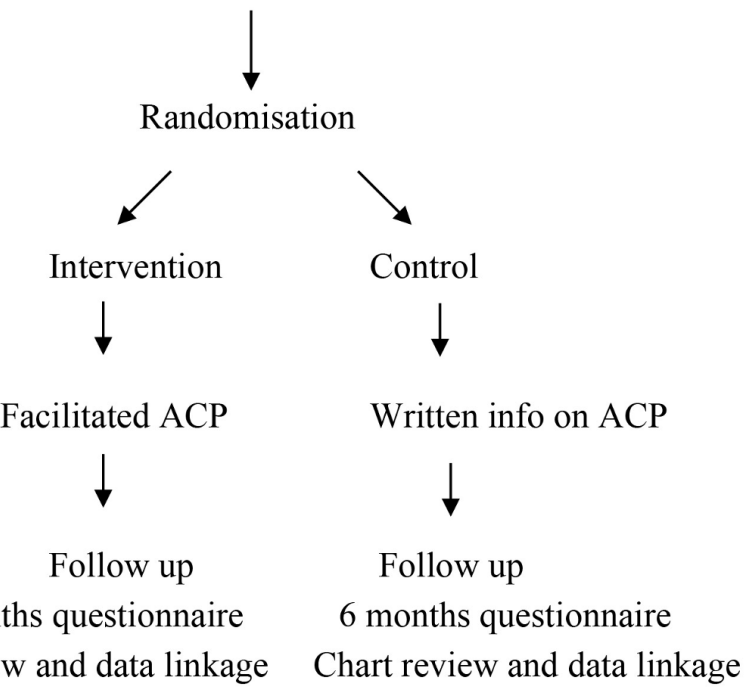

*If Supportive and Palliative Care Indicators Tool (SPICT) reveals that the patient has dementia or other illnesses that may impact on the patient's decision-making capacity, then the ACP Coordinator will confer with the treating medical team to determine if they have the decision-making capacity to provide informed consent. If they do not, then the person responsible will be invited to provide informed consent, together with the patient.

Figure 1 Participant assessment and follow-up plan. ACP, advance care planning.

documents, such as uploading them to the hospital electronic medical record system. The model of ACP used in the study has been in active clinical use for many years at several hospital sites that are taking part in the current study. It has been refined through active clinical practice, is approved by the hospital management and is widely accepted by the clinicians and patients.

Registered nurses from aged care, palliative care and chronic disease management background with interest and expertise in care coordination and ACP have been recruited as ACP coordinators for the project. They received face-to-face training in ACP and will be supervised by a Chief Investigator (AM) who is an expert in ACP and has conducted many training workshops. The three ACP coordinators will work closely with the staff of the participating outpatient clinics and will be responsible for initiating and conducting ACP discussions, answering questions from patients, caregivers and staff and assisting the patients with completion of ACDs and other documents. They will organise and facilitate meetings and teleconferences with clinical staff (eg, specialists and GPs) involved in the care of the patient and assist with dissemination of the completed documents using the hospital electronic medical records system and to GPs and specialists. The ACP coordinators will also provide education, training and mentorship to clinic staff, GPs and other health providers and help them incorporate ACP into routine care. The ACP coordinators will not have any other involvement in patient care.

\section{Clinical staff ACP training}

The clinical staff (medical, nursing, allied health) working in the participating outpatient clinics will receive education and training in ACP through lunchtime seminars, workshops or unit meetings run by members of the research team and the project ACP coordinators. The training will use existing resources, such as Planning Ahead website (http://planningaheadtools.com.au/), NSW Health endorsed ACP booklet, ${ }^{18}$ NSW Health documents and directives, such as the Using Resuscitation Plans in End 
Table 1 Elements of ACP interventions in RCT

\section{Intervention group (facilitated ACP)}

Written information on ACP:

- ACP brochure produced by NSW Health ${ }^{18}$

- ACP workbook endorsed by NSW Health ${ }^{19}$

- Enduring Guardianship NSW booklet produced by the Public Guardian Office of the NSW Government Department of Attorney General \& Justice ${ }^{20}$

- Statement of Values and Wishes completed on behalf of the patient by their person responsible (when the person is unable to develop an ACD) and supplemental information for families

Procedures, activities, processes delivered by ACP

coordinators or ACP trained outpatient clinics staff:

- Provision of personalised information on ACP

- Facilitation of ACP discussions with patients, caregivers, family and relevant health professionals

- Assistance in completing appropriate ACP documents

- Referral to relevant local legal services for witnessing as required

- Organising and facilitating meetings and/or teleconferences with clinic staff, GPs and other healthcare professionals involved in patient's care to discuss the patient's care and their wishes

- Assistance with dissemination of ACP documents on completion that may include upload to LHD record system, national eHealth record (contingent on LHD and national eHealth improvements during the study period) and forwarding the document to patient's GP

\section{Control group (current standard care)}

Written information on ACP:

- ACP brochure produced by NSW Health ${ }^{18}$

- ACP workbook endorsed by NSW Health ${ }^{19}$

- Enduring Guardianship NSW booklet produced by the Public Guardian Office of the NSW Government Department of Attorney General \& Justice 20

- Statement of Values and Wishes and supplemental information for families used for families to develop a plan (when the person is unable to develop an ACD)

ACP, advance care planning; LHD, Local Health District; NSW, New South Wales; RCT, randomised controlled trial.

of Life Decisions document, ${ }^{21}$ and Health Education and Training Institute (HETI) training modules. Additional topics covered in the training will include legal liability and protection offered by Advance Care Plans and ACDs, capacity determination and hierarchy of decision-making in NSW.

Both the clinical staff training and the facilitated ACP intervention are informed by the Diffusion of Innovation Theory. ${ }^{22}$ This theory states that five conditions need to be met for innovation to be taken up: relative advantage, compatibility, complexity (how difficult the innovation appears), trialability and observability (whether positive outcomes of the innovation can be observed). This means that didactic education sessions alone are unlikely to be sufficient to lead to significant increase in ACP undertaken by health professionals. For instance, compatibility with clinical service workflow or positive impact of ACP may not be readily apparent to busy clinicians, until they have experienced first-hand, ACP done with their patients. The study intervention allows staff members of clinical services to 'see' a few and 'do' a few, thereby increasing the chance of ACP being embedded into the routine care of patients.

Clinic staff will be initially invited to join ACP sessions facilitated by ACP coordinators (ie, 'see') in their clinical role (eg, as a doctor or nurse participant). After a few observations, clinic staff will be invited to facilitate ACP discussions (ie, 'do') with ACP coordinators observing and providing support. And finally, the clinic staff will conduct sessions independently with ACP coordinators providing telephone and email support. The precise details of how many clinic staff members undertake 'see' and 'do' will vary from clinic to clinic according to their staffing levels and available resources.

In addition, the research team will facilitate educational sessions for local GPs and other health professionals providing care for the patients enrolled in the study, in conjunction with the Central and Eastern Sydney Primary Health Network, an important partner in the study. ACP coordinators will also use their interactions with the patient's GP or other health providers involved in the patient's care as opportunities to promote ACP and for education. For instance, when they fax a copy of the patient's ACD to the GP, this will be accompanied by an information sheet on ACP and the web address of the Planning Ahead website (http://planningaheadtools.com. $\mathrm{au})$.

\section{Participant recruitment and enrolment}

The outpatient clinic staff, either independently or with assistance from the ACP coordinators, will apply the SPICT $^{16}$ to the patients attending the clinic that day. Having two or more general indicators, or one or more specific clinical indicators, will be taken as satisfying the 
SPICT criteria. Patients identified as meeting the SPICT criteria will be handed a 1-page brochure with information about the project by the clinic staff on their arrival to the clinic appointment, unless the treating clinical team felt that it would be inappropriate to invite them to the study. Those expressing an interest in the study will be invited to meet with the ACP coordinator who will administer the ACP screening tool ${ }^{17}$ to assess whether a patient had previously been involved in ACP and if not then whether they are interested in engaging in ACP. Patients who had not been involved in ACP and were interested in ACP will be provided with further information about the study and invited to provide written informed consent. The ACP coordinator will check the inclusion and exclusion criteria to make sure that the patient was eligible to participate in the trial. If SPICT reveals that the patient has dementia or other illness that may impact on the patient's decision-making capacity, the treating medical team will be asked to determine if the patient has sufficient decision-making capacity to provide informed consent. If they do not, the person responsible will be invited to provide informed consent.

A participant will be enrolled into the study after the informed consent has been obtained and if the participant meets all inclusion criteria and does not meet any of the exclusion criteria. The participant will then complete a baseline patient questionnaire and nominate a caregiver to complete a baseline caregiver questionnaire. Following the completion of the enrolment questionnaires, the patient will be randomised to a control or intervention group.

\section{Primary outcome}

The primary outcome is the number of unplanned hospital admissions, defined as admissions or readmissions with an overnight stay that were not previously planned or scheduled or 'elective', ${ }^{23}$ during the 6-month follow-up period. The data will be collected via an audit of the hospital records after the 6-month follow-up period.

\section{Secondary outcomes}

Several secondary outcomes will be assessed at the 6-month follow-up. The data will be collected using self-report questionnaires, audit of hospital records, audit of ACP documentation and data linkage analysis (table 2).

Secondary outcomes assessed via self-reported surveys:

- Patient's health related quality of life (SF-20).

- Patient's reported quality of chronic disease care (PACIC).

- Caregiver's health related quality of life (SF-20).

- Primary caregiver burden (ZBI-12).

- Clinical staff's knowledge and attitudes to ACP.

Secondary outcomes measured using hospital records audits:

- Hospital admissions (unplanned and planned).

- ED presentations.

- Cardiopulmonary resuscitation (CPR) attempts.
- Documentation of ACDs and patient wishes in patient records.

Secondary outcomes measured using data linkage analysis:

- Ambulance services calls.

- ED presentations.

- Total number of hospital admissions.

- Date, place and causes of death.

- Resuscitation attempts.

- Intensive care unit admissions.

\section{Data collection and follow-up}

Screening

Patient's suitability for participation in the RCT will be assessed using the SPICT ${ }^{16}$ and the ACP Screening Interview adapted from an original interview developed by Cheang et al. ${ }^{17}$ SPICT is a widely used tool that allows health and social care professionals to identify patients with advanced illnesses, who are potentially having supportive and palliative care needs and are at higher risk of death in the next 6-12 months. SPICT has six general indicators of deteriorating health and clinical signs of advanced illnesses, such as congestive cardiac failure, chronic advanced respiratory illness that causes dyspnoea at rest or requires home oxygen, end stage renal failure, especially where dialysis has been stopped, advanced cirrhosis with complications such as hepatorenal syndrome and frailty. The modified version of the ACP Screening Interview will be used to determine whether patients have already been involved in ACP and if they have not, then whether they are interested in engaging in ACP.

\section{Baseline data collection}

The baseline enrolment questionnaire includes the patient's basic demographic information including age, gender, marital status, employment status, country of birth, language spoken at home, Aboriginal or Torres Strait Islander heritage, level of education and whether they had a regular general practitioner. The patient's caregiver will provide their own basic demographics data (age, gender, marital status, employment status, country of birth, language spoken at home, Aboriginal or Torres Strait Islander heritage and level of education) and the number of hours per week spent on caregiving duties. The clinic staff member will complete the Charlson Comorbidity Index (CCI ${ }^{24}$ for the patient. The CCI is a validated measure of 1-year mortality risk and burden of disease, feasible in various healthcare settings. It consists of 17 comorbidities, including two subcategories for diabetes and liver disease, which are weighted from 1 to 6 for mortality risk and disease severity and summed to the total CCI score.

Also at baseline as well as at the 6-month follow-up, self-report patient data will be collected on the health-related quality of life using the 20-Item Short Form Health Survey (SF-20) ${ }^{25} 26$ and the quality of chronic disease care using the Patient Assessment of Chronic Illness Care (PACIC). ${ }^{27}$ The SF-20 is a validated measure of 
Table 2 Study outcomes/variables, measurement tools and data collection schedule

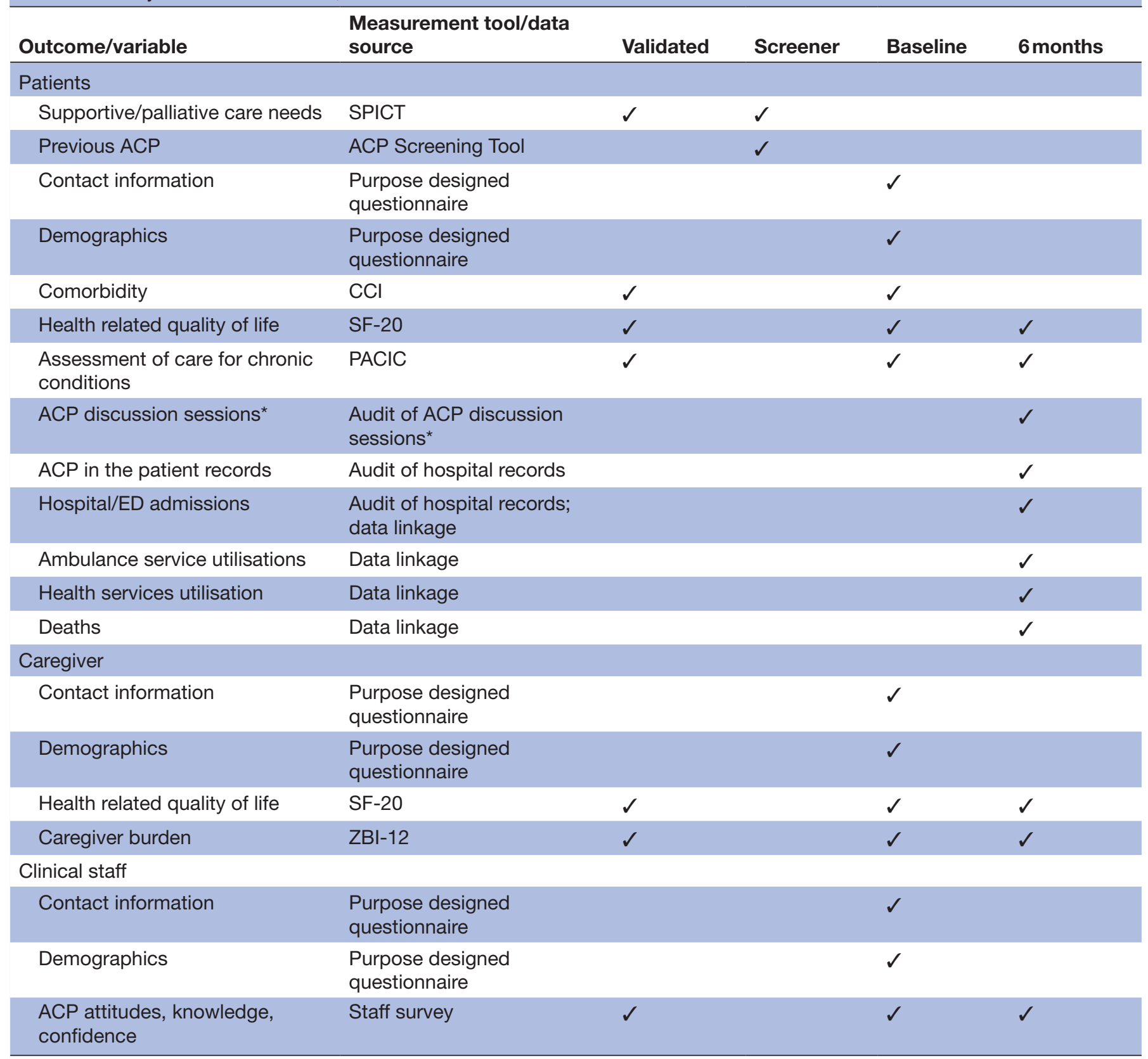

${ }^{*}$ Intervention group only; CCl: Charlson Comorbidity Index; ${ }^{23}$ PACIC: Patient Assessment of Chronic Illness Care; ${ }^{26}$ SF-20: $20-$ Item Short Form Health Survey; ${ }^{24}$ SPICT: Supportive and Palliative Care Indicators Tool; ${ }^{15}$ ZBI-12: Zarit Burden Short Form Interview. ${ }^{27}$

ACP, advance care planning; ED, emergency department.

multiple health dimensions: physical functioning, role functioning, social functioning, mental health, perceptions of current health and pain. The SF-20 scores are transformed linearly to $0-100$ scales, where 0 is the lowest possible score and 100, the highest possible score. The PACIC is a validated 20-item instrument to assess chronic illness patient's experience in five domains of care: patient activation, delivery system design, goal setting, problem solving and follow-up/coordination. The score is obtained by summing participants' responses across all 20 items on a 5-point Likert scale, divided by 20. PACIC total scores range from 1 to 5 ; higher scores indicate patient's perception of greater involvement in self-management and chronic care counselling.

Caregivers of patients will be asked to complete a self-report questionnaire focusing on their quality of life (SF-20) and caregiver burden (ZBI-12), ${ }^{28}$ at baseline and at the 6-month follow-up. The ZBI-12 is a validated instrument to provide brief and accurate assessment of caregiver burden. Each ZBI-12 item is a statement to be endorsed on a 5-point Likert scale (0-never, 4-nearly always); a higher score indicates a higher level of caregiver burden. 
Clinical staff working in the outpatient clinics and participating in the ACP training will complete presurveys and postsurveys on ACP knowledge and attitudes. The survey consists of nine ACP knowledge items and nine items measuring beliefs, attitudes, experiences and perceived confidence and willingness of clinical staff to conduct ACP. The survey items were designed by a registered nurse and a GP with expertise in ACP and primary palliative care and tested in a study looking at ACP confidence levels and motivation in practice nurses in a GP setting. ${ }^{29}$ The knowledge questions involve 'yes/no' questions and multiple choice questions and the attitude items are based on a five-point Likert scale with levels of agreement ranging 'strongly disagree' to 'strongly agree'.

\section{6-month data collection}

At the 6-month follow-up, self-report patient data will be collected on the health-related quality of life (SF-20 $)^{2526}$ and the quality of chronic disease care (PACIC). ${ }^{27}$ Caregivers of patients will be asked to complete a self-report questionnaire focusing on their quality of life (SF-20) and caregiver burden (ZBI-12) ${ }^{28}$ Clinical staff working in the outpatient clinics and participating in the ACP training will complete a 6-month follow-up survey on ACP knowledge and attitudes. ${ }^{29}$

The 6-month follow-up involves an audit of ACDs (both intervention and control group) and an audit of ACP discussion sessions (intervention group only). The audit of ACD measures the output of ACP as indicated by the number and types of documents created as a result of ACP discussions. The audit of ACP discussion sessions involves collection of data on time taken; number of attendees and their categories (eg, patient, facilitator, family member, other healthcare professionals); number and types of documents completed; number and types of documents distributed; recommendations made to appoint substitute decision makers and referrals made to legal and specialist services.

The 6-month follow-up hospital records audits will collect data on planned and unplanned hospital admissions, ED presentations, admission into the intensive care unit, cardiopulmonary resuscitation, major procedures and investigations, documentation of ACDs and patient wishes in patient records. Additionally, the following information will be collected through data linkage in collaboration with the Centre for Health Record Linkage (CHeReL): (1) ambulance services calls assessed by data linkage to NSW Ambulance Data Collection, (2) ED presentations assessed by data linkage to NSW Emergency Department Data Collection, (3) total number of hospital admissions assessed by data linkage to NSW Admitted Patient Data Collection, (4) date, place and causes of death assessed by data linkage NSW Mortality Data, 5) resuscitation attempts assessed by data linkage to NSW Emergency Department Data Collection, NSW Admitted Patient Data Collection, NSW Ambulance Data Collection and (6) Intensive Care Unit admissions assessed by data linkage to NSW Admitted Patient Data Collection.
At the 6-months mark, a small number of participants including patients, caregivers and healthcare professionals, will be invited to participate in semistructured interviews and focus groups. The interviews and focus groups will explore the acceptability and feasibility of the facilitated ACP process for patients, caregivers and clinical staff. The study will also evaluate the ACD form and the accompanying information booklet used in the study. This evaluation will canvass the views of patients, their caregivers and health professionals about the usefulness and appropriateness of these documents as well as their recommendations for improving the template.

\section{Sample size}

The sample size calculation is based on an assumed alpha of 0.05 , power of 0.8 to detect at least $25 \%$ reduction (IRR $0.75)$ in unplanned hospital admissions over 6 months (from an average of 2.2 over 6 months to 1.65 admissions over 6 months assuming the number of admissions follows a Poisson distribution in each group). Based on these assumptions, the study needs to recruit 100 people in each group, who will have the full 6-month follow-up, so that the number of hospitalisations in the groups are 220 (control) and 165 (intervention). However, given that the expected death rate in the 6 months of the trial is up to $35 \%$, the study needs to recruit 122 people in each arm of the trial. If the expected drop-out rate (withdrawal, loss to follow-up) is $15 \%, 144$ people are needed in each arm of the trial (in total $n=288$ ).

The estimated mean numbers of hospital admissions were obtained from a study conducted in Scotland ${ }^{16}$ which showed that patients with advanced renal, liver, cardiac and respiratory conditions identified by SPICT as at risk of dying within 12 months had high hospital unplanned admission rates in the next 6 months (for patients alive, mean 2.2 admissions in 6 months; for patients who have died, mean 2.4 admissions in last 6 months of life). This study also showed a death rate of $35 \%$ over a 6 -month period. Effect size estimates for ACP in reducing admissions are based on a study by Caplan et $a l^{6}{ }^{6}$ which showed that patients from nursing homes that had ACP programme had relative risk of hospital admission of 0.74 compared with control homes. Molloy et a $\vec{l}^{7}$ conducted a cluster RCT in Canadian nursing homes that showed that patients in nursing homes with ACP had mean admission rate of 0.27 per resident, compared with 0.48 for control homes over 18-month period. The effect size estimate of at least $25 \%$ reduction in admissions over 6 months is reasonable when compared with these figures.

\section{Randomisation}

Prior to randomisation, the patient participants are required to meet all the inclusion criteria and do not meet any of the exclusion criteria, screened using the ACP screening tool, provide written informed consent and complete the baseline questionnaire. Patients are randomised to either intervention or control group using an online randomisation service (randomize.net). Randomisation is at the individual level by a computer generated random number sequence, in blocks of random sizes (4 and 6), in 1:1 ratio (control and 
intervention). Stratification is according to the study site (hospital) and the number of hospital admissions in the past 6 months ( $<2$ vs 2 or more).

Study conducted by Highet et $a l^{16}$ showed significant differences in unplanned admission rates and death rates in patients who had $<2$ admissions in the 6 months prior to the study period compared with those who had 2 or more admissions. Due to the nature of the intervention, it is not possible to blind the patients participating in the study and clinical staff providing the ACP intervention, including ACP coordinators.

\section{Data analysis}

\section{Quantitative data}

Descriptive statistics will be presented as proportions or means, with SD. Statistically significant difference in means between the intervention and control groups for continuous variables will be analysed using tests such as student's t-test and analysis of variance. $\chi^{2}$ and similar tests will be used to test statistical significance of categorical variables. Multivariate regression will be used to analyse the impact that independent variables may have on primary and secondary outcomes.

For the primary outcome (ie, number of unplanned hospital admissions during at 6-month follow-up), a Poisson regression model will be fitted, including follow-up time as an offset to compare the mean number of admissions between the trial arms. The analysis will also adjust for the variables used in stratification in the randomisation (number of admissions in 6 months prior to the trial and the study site). Secondary outcomes at 6 months, such as ambulance service calls, ED presentations, hospital admissions and other patient outcomes, will be analysed similarly to the primary outcome. Linear regression adjusting for stratification variables will be used to analyse other secondary outcomes at 6 months, such as health related quality of life, caregiver burden and assessment of quality of chronic disease care. Descriptive statistics will be used to analyse frequency of documentation of patient wishes in patient records, in the audit of SPICT, ACP screening tool forms completed by clinic staff and the ACDs and advance care plans completed during the study. Analysis of the results will be based on the initial group allocation, regardless of whether the intervention has been delivered in intervention participants or in the case of control group participants, whether they have engaged in ACP.

\section{Patient and public involvement}

The development of the research question and outcome measures was informed by the findings of the literature outlining the potential benefits that ACP can have on patients and caregivers. The study therefore includes multiple patient reported outcome measures as well as similar measures for the caregivers as well as a qualitative substudy to capture the experience of the patients and caregivers of being in the intervention and the control group.

Representatives from consumer organisations and patient advocacy groups are actively involved in the study steering group and have provided input into the study design and the conduct of the study.

A summary of the study findings will be provided to the study participants.

\section{Ethics and dissemination}

Ethics approval was obtained from the SESLHD Human Research Ethics Committee (HREC/16/POWH/654) and the NSW Population \& Health Services Research Ethics Committee (2017/HRE1103). The trial has been registered with the Australian and New Zealand Clinical Trials Registry (Trial ID: ACTRN12617000280303). The study was approved by the NSW Population and Health Service Research Ethics Committee (2017/HRE1103) for the use of linked data owned or managed by NSW Ministry of Health, including the NSW Admitted Patient Data Collection and the NSW Emergency Department Data Collection.

Study results will be disseminated via reports to the funding body, academic presentations at conferences, journal publications and seminar sessions, workshops and invited talks.

\section{DISCUSSION}

The current study has been designed to determine whether a model of facilitated ACP intervention for patients with advanced illnesses attending hospital outpatient clinics can reduce unplanned hospital admissions and acute health services usage and improve the quality of care for patients and their caregivers/family. This study has several strengths and limitations which are presented below.

\section{Strengths}

This RCT has a number of potential benefits. It may provide high-level (RCT) evidence that ACP conducted outside of the residential care home setting can lead to reduced unplanned hospital admissions and acute health services usage. Importantly, it could provide evidence that this can be achieved while at the same time improving patient quality of life, satisfaction with care and reducing caregiver burden. Further, the proposed model of ACP has the potential to incorporate ACP into routine care of patients with advanced illnesses in outpatient clinics and by other health professionals involved in their care. This will be done by a process of diffusion, for instance, clinic staff are initially invited as participants in ACP discussions facilitated by trained ACP coordinators and as they gain experience and receive training and education, they facilitate ACP independently. The delivery of the facilitated ACP intervention requires ongoing involvement of skilled clinical staff in a busy everyday clinical practice. Training and support, informed by the Diffusion of Innovation Theory and provided in the study by the ACP coordinators in clinical settings, should ensure quality and fidelity of the delivered intervention.

\section{Limitations}

Significant drop-out rate due to withdrawal and/or loss to follow-up, and high mortality, is to be expected in an 
intervention study of patients with advanced illnesses. This limitation has been taken into account in the calculation of an adequate sample size. In addition, the study excludes people whose English language skills are not adequate as documents were only available in English.

\section{Trial status}

Recruitment for the study commenced in April 2017 and was completed in December 2017. The interventions were conducted between May 2017 and December 2017. The 6-month follow-up (patient and caregiver questionnaires, audits) is expected to be completed by July 2018, with the data linkage extraction and analysis taking place in late 2018.

\section{Author affiliations}

${ }^{1}$ School of Medicine, University of Wollongong, Wollongong, New South Wales, Australia

${ }^{2}$ Centre for Primary Health Care and Equity, University of New South Wales, Sydney, New South Wales, Australia

${ }^{3}$ Post Acute Care Services, Prince of Wales Hospital, South Eastern Sydney, Sydney, New South Wales, Australia

${ }^{4}$ Southcare, Sutherland Hospital, South Eastern Sydney, Sydney, New South Wales, Australia

${ }^{5}$ Centre for Education and Research on Ageing, Concord Clinical School, University of Sydney and Ageing and Alzheimer's Institute, Concord Repatriation General Hospital, Sydney, New South Wales, Australia

${ }^{6}$ Faculty of Health Sciences and Medicine, Bond University, Gold Coast, Queensland, Australia

${ }^{7}$ Australian Centre for Public and Population Health Research, University of Technology, Sydney, New South Wales, Australia

${ }^{8}$ Department of Geriatric Medicine, Concord Hospital, Sydney, New South Wales, Australia

${ }^{9}$ Aged, Chronic Care \& Rehabilitation, Sydney Local Health District, Sydney, New South Wales, Australia

${ }^{10}$ Department of Geriatric Medicine, Prince of Wales Hospital, Sydney, New South Wales, Australia

Contributors JR, AM, GAC, PG and NZ conceived the study. JR, AM, GAC, PG, VN, $\mathrm{NZ}, \mathrm{AH}, \mathrm{JC}$ and J-AOK wrote the study protocol. AH designed the statistical analysis plan for the protocol. JR, AM and KK designed the implementation aspects of the protocol. JMcD and BH-R made substantial contributions to the study design. KK and JR were responsible for drafting the manuscript. All authors read, provided important revisions and approved the final version of the manuscript.

Funding This work was supported by the NSW Ministry of Health: Translational Research Grant number 143

Competing interests None declared.

Patient consent for publication Not required.

Ethics approval South-East Sydney Local Health District Human Research Ethics Committee.

Provenance and peer review Not commissioned; externally peer reviewed.

Open access This is an open access article distributed in accordance with the Creative Commons Attribution Non Commercial (CC BY-NC 4.0) license, which permits others to distribute, remix, adapt, build upon this work non-commercially, and license their derivative works on different terms, provided the original work is properly cited, appropriate credit is given, any changes made indicated, and the use is non-commercial. See: http://creativecommons.org/licenses/by-nc/4.0/.

\section{REFERENCES}

1. Cartwright CM, Parker MH. Advance care planning and end of life decision making. Aust Fam Physician 2004;33:815-9.

2. Tulsky JA. Beyond advance directives: importance of communication skills at the end of life. JAMA 2005;294:359-65.
3. Kirsner S. Advance care planning and quality improvement. Aust $J$ Prim Health 2008;14:89-95.

4. Detering KM, Hancock AD, Reade MC, et al. The impact of advance care planning on end of life care in elderly patients: randomised controlled trial. BMJ 2010;340:c1345.

5. Chan HY, Pang SM. Let me talk-an advance care planning programme for frail nursing home residents. J Clin Nurs 2010;19:3073-84.

6. Caplan GA, Meller A, Squires B, et al. Advance care planning and hospital in the nursing home. Age Ageing 2006;35:581-5.

7. Molloy DW, Guyatt GH, Russo R, et al. Systematic implementation of an advance directive program in nursing homes: a randomized controlled trial. JAMA 2000;283:1437-44

8. Houben CHM, Spruit MA, Groenen MTJ, et al. Efficacy of advance care planning: a systematic review and meta-analysis. J Am Med Dir Assoc 2014;15:477-89.

9. National Health and Hospitals Reform Commission. A healthier future for all Australians - final report of the National Health and Hospitals Reform Commission. Canberra: National Health and Hospitals Reform Commission, 2009.

10. NSW Ministry of Health. Advance care planning for quality care at end of life. Action plan 2013-2018.: NSW Ministry of Health, 2013.

11. Rhee JJ, Zwar NA, Kemp LA. Uptake and implementation of advance care planning in Australia: findings of key informant interviews. Aust Health Rev 2012;36:98-104.

12. Zib M, Saul P. A pilot audit of the process of end-of-life decisionmaking in the intensive care unit. Crit Care Resusc 2007;9:213-8.

13. Chan DK, Ong B, Zhang K, et al. Hospitalisation, care plans and not for resuscitation orders in older people in the last year of life. Age Ageing 2003;32:445-9.

14. White $\mathrm{B}$, Tilse $\mathrm{C}$, Wilson $\mathrm{J}$, et al. Prevalence and predictors of advance directives in Australia. Intern Med J 2014;44:975-80.

15. Ditto PH, Jacobson JA, Smucker WD, et al. Context changes choices: a prospective study of the effects of hospitalization on life-sustaining treatment preferences. Med Decis Making 2006;26:313-22.

16. Highet G, Crawford D, Murray SA, et al. Development and evaluation of the Supportive and Palliative Care Indicators Tool (SPICT): a mixed-methods study. BMJ Support Palliat Care 2014;4:285-90.

17. Cheang F, Finnegan T, Stewart C, et al. Single-centre cross-sectional analysis of advance care planning among elderly inpatients. Intern Med J 2014:44:967-74.

18. NSW Health. Advance care planning: making your wishes known. 2016 http://www.health.nsw.gov.au/patients/acp/Documents/ making-your-wishes-known.pdf

19. NSW Health. Making an advance care directive: form and information booklet. 2017 http://www.health.nsw.gov.au/patients/acp/Pages/ acd-form-info-book.aspx

20. Public Guardian Office of the NSW Government Department of Attorney General \& Justice. Enduring Guardianship in New South Wales. Your way to plan ahead. 2014

21. NSW Ministry of Health. Using resuscitation plans in end of life decisions. Policy directive. 2014.

22. Sanson-Fisher RW. Diffusion of innovation theory for clinical change. Med J Aust 2004:180:S55.

23. Purdy S, Paranjothy S, Huntley $\mathrm{A}$, et al. Interventions to reduce unplanned hospital admission. Bristol: A series of systematic reviews, 2012.

24. Charlson ME, Pompei P, Ales KL, et al. A new method of classifying prognostic comorbidity in longitudinal studies: development and validation. J Chronic Dis 1987;40:373-83.

25. Stewart AL, Hays RD, Ware JE. The MOS short-form general health survey. Reliability and validity in a patient population. Med Care 1988;26:724-35.

26. Ware JE, Sherbourne CD, Davies AR. Developing and testing the MOS 20-item short-form health survey: A general population application. In: Stewart AL, Ware JE, edn. measuring functioning and well-being: the medical outcomes study approach. Durham, NC: Duke University Press, 1992:277-90.

27. Glasgow RE, Wagner EH, Schaefer J, et al. Development and validation of the Patient Assessment of Chronic Illness Care (PACIC). Med Care 2005;43:436-44.

28. Bédard M, Molloy DW, Squire L, et al. The Zarit Burden Interview: a new short version and screening version. Gerontologist 2001;41:652-7.

29. Fan E, Rhee JJ. A self-reported survey on the confidence levels and motivation of New South Wales practice nurses on conducting advance-care planning (ACP) initiatives in the general-practice setting. Aust J Prim Health 2017;23:80-6. 